OPEN ACCESS

Edited by:

Ankur Gogoi,

Jagannath Barooah College, India

Reviewed by:

Mehmet Burcin Unlu,

Boğaziçi University, Turkey

Stefano Pagliara,

University of Exeter, United Kingdom

${ }^{*}$ Correspondence:

Ellas Spyratou

ellas5@central.ntua.gr

Specialty section:

This article was submitted to

Optics and Photonics,

a section of the journal

Frontiers in Physics

Received: 09 November 2021 Accepted: 03 February 2022

Published: 22 February 2022

Citation:

Spyratou E (2022) Advanced Biophotonics Techniques: The Role of Optical Tweezers for Cells and Molecules Manipulation Associated With Cancer.

Front. Phys. 10:812192. doi: 10.3389/fphy.2022.812192

\section{Advanced Biophotonics Techniques: The Role of Optical Tweezers for Cells and Molecules Manipulation Associated With Cancer}

\author{
Ellas Spyratou * \\ Department of Medicine, Democritus University of Thrace, Alexandroupolis, Greece
}

Rapid advances in Biophotonics are revolutionizing the illumination of several diseases and, among them, the monitoring of cancer pathogenesis and therapy. Today, several efforts aim to miniaturize the Biophotonics tools, leading to the namely Nanobiophotonics. This scientific field refer to the development of novel technologies, biosensors, and drug delivery systems for prevention, diagnosis, and treatment of diseases at the nanoscale, in sub-cellular and molecular level. Modern non-invasive laser-based techniques are applied in different domains, from practical, clinical applications to molecular and cellular biology fundamental research. Among the plethora of photon-based techniques, optical trapping is a very promising tool for improving the understanding of cancer at cellular level. Recently, optical tweezers are revived as a potential technique for cell characterization, tracking cells behavior and probing interactions forces between cells, cells-biomolecules, and cellsnanoparticles. In this review, we aim to exhibit the state-of the art advances of Biophotonics in the diagnostic and therapeutic field of cancer focusing on the role of optical tweezers.

Keywords: biophotonic techniques, optical tweezers, cancer diagnosis, cancer therapy, cells characterization

\section{INTRODUCTION}

Biophotonics is an emerging multidisciplinary research area, embracing all light-based technologies applied to the life sciences and medicine. The expression itself is the combination of the Greek syllables' "bios" standing for life and "phos" standing for light [1]. In Biophotonics, the "conventional" light is monochromatic laser or laser-like non-ionizing radiation and the basic biomedical applications to all levels of biological structures are divided into two major fields. The first is devoted to diagnostic and imaging applications (in vivo and in vitro, in cellular or molecular level) and the second to therapy or surgery using photon radiation (e.g., biostimulation, tissue removalsurgery, photodynamic therapy, cell micromanipulation) [2]. Modern non-invasive laser-based optical research techniques prove to be more and more useful in the biomedical field, covering very different domains, from practical, clinical applications to molecular and cellular biology fundamental research $[3,4]$.

Today, several efforts aim to the miniaturization of Biophotonics tools, leading to Nanobiophotonics [5, 6]. This advanced scientific field refers to the research and development of novel technologies, biosensors, and drug delivery systems for prevention, diagnosis, and treatment of diseases at the nanoscale, in sub-cellular and molecular level and for the dream of personalized therapy [7]. Moreover, laser-based techniques and instrumentation have driven to a new era in cell 
biology, the intracellular nanosurgery [8]. This technology has allowed the ability to perform precise nano-incisions in cells and manipulation of intracellular structures or even at the level of individual genes within the nucleus [9]. Laser nano-surgery combined with monitoring devices can lead from intracellular ablations to in vivo subcellular dissections [10, 11].

Among the plethora of photon-based techniques, optical tweezers, with the ability of applied Biophotonics interventions in living cells, is a very promising tool in cancer field [12, 13]. Optical tweezers technique is a non-invasive biomedical tool with advanced applications in biology [14], medicine [15], and nanotechnology [16]. The ability to "touch" the microcosmos non-invasively, while performing nanometer-precision and submicrometric analysis, using a single optical tool, is a revolutionary technique. Optical tweezers can manipulate cells, viruses, bacteria and macromolecules. Using the optical trapping technique, a cell can be selectively, non-invasively and nondestructively manipulated to a phagocyte, attached to its surface cell receptor and trigger the initiation of the phagocytosis process [17]. Optical tweezers are capable to grab, tracking and manipulate small virus such as influenza [18]. This review highlights the novel photon-based theranostics modalities for cancer confrontation focusing on the role of optical tweezers. The advanced applications of optical tweezers in biology and Nanomedicine are presented. New potential prospects of the optical tweezers in the cancer field are also provided.

\section{BIOPHOTONICS AND THERANOSTICS IN CANCER}

\section{Biophotonics in Diagnosis of Cancer}

Biophotonics is a relatively novel interdisciplinary discipline that integrates lasers, optoelectronics, photonics and biomedical sciences, dealing with the interaction between non-ionizing light quanta and biological materials, including tissues, cells and even sub-cellular structures and molecules in living organisms [19]. In the literature, there are a variety of research and clinical studies based on cellular and sub-cellular diagnosis via flow cytometry [20], light-microscopy techniques and laser-induced fluorescence spectroscopy such as: Epifluorescence microscopy [21], immunofluorescence microscopy [22], optical coherence tomography [23], confocal microscopy [24], Total Internal Reflection Fluorescence - TIRF microscopy [25], Two-Photon Laser Scanning Microscopy-TPLSM [26], Fluorescent Resonance Energy Transfer-FRET [27], PhotoActivated Localization Microscopy - PALM [28], nanolaser confocal spectroscopy [29]. These techniques enable real-time and/or in situ imaging of living tissue at high resolution and high contrast, without physically dissecting the tissue. These imaging techniques can find great applications in Nanomedicine.

Multiple imaging modes can offer complementary information and overcome the limitations of each single modality. The combination of optical imaging with computed tomography (CT), magnetic resonance imaging (MRI), Positron
Emission Tomography (PET) and Single-photon emission computed tomography (SPECT) can enhance size resolution and penetration depth [30]. Near-Infrared Fluorescence (NIRF) imaging is highly attractive for early non-invasive detection of cancer due to its high penetration depth and low autofluorescence [31]. Different types of multicomponent nanoparticles like PEGylated $\mathrm{Au} / \mathrm{SiO} 2$ nanocomposites conjugated with Fluorescein isothiocyanate (FITC) [32], Iron Oxide NPs encapsulating in Human Serum Albumin (HAS) [33] etc. have been designed to act as dual contrast agents offering multimodal imaging (Figure 1). The combination of CT, MR, PET and SPECT modalities with fluorescence imaging modalities can allow extension of imaging across the dynamic range of size resolution and penetration depth from deep-body with size resolution of $\sim 1 \mathrm{~mm}$ to thin penetration depths of few hundred microns or millimeters with size resolutions to singlecell or even subcellular resolution [34-36].

In recent years, the biophotonics techniques have been integrated with machine learning methodologies based on artificial neural networks (ANNs) [37-39]. This will give a boost in the Biophotonic fields to obtain real-time decisionmaking systems for doctors, biologists, pathologist etc. by analyzing a large datasets of image data and spectral data [37]. For example, ANNs were used to diagnose tongue squamous cell carcinoma based on the difference in Raman Spectral signature between healthy and malignant cells for the accurate intraoperative discrimination between healthy and cancerous margins [40]. Figure 2 shows a schematic image of the workflow of the combination of Raman spectroscopy with machine learning models for tissue discrimination. ANNs have been applied to Scanning Enhanced Raman Spectroscopy optophysiology data to probe metabolite gradients in a variety of cell lines such as HeLa and HUVEC [41]. Machine learning models have been created to identify DNA damage in a nasopharyngeal carcinoma cell line (CNE2) after x-ray radiation [42] from spectral data obtain by silver nanoparticle-based surfaceenhanced Raman scattering (SERS) [43]. Deep learning models have been applied in $\gamma$-H2AX immunofluorescence images to quantify the number of $\gamma$-H2AX foci for the detection of DNA double-strand breaks. The $\gamma-\mathrm{H} 2 \mathrm{AX}$ foci is a sensitive biomarker for the quantification of the DNA damage [44]. These foci are formed specifically at sites of DNA double-strand breaks after ionizing radiation exposure as a cellular response of the lymphocytes in peripheral blood [45, 46].

\section{Biophotonics in Therapeutics of Cancer}

Recent theranostics techniques are combined with nano-imaging and nanomaterial-based drug delivery techniques for an effective and targeted disease management [47-49]. Metallic nanoparticles, semiconductor quantum dots and carbon nanotubes have been used as photosensitizer agents for phototriggered diagnosis and photo-triggered therapy [50, 51]. Antibodies, peptides and small molecules can be labeled with fluorescence dyes and conjugated to nanoparticles producing targeted optical imaging probes for cancer detection [52, 53]. Nanocarriers can be served as multi-modal theranostics systems [54] or as "Trojan Horses" [55] carrying multiple therapeutic and 
A

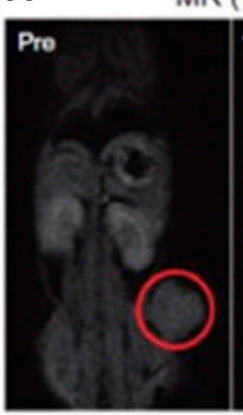

B NIRF

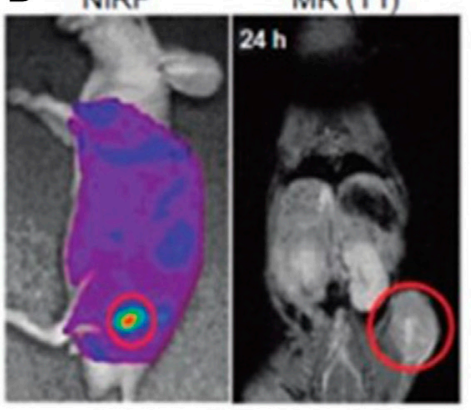

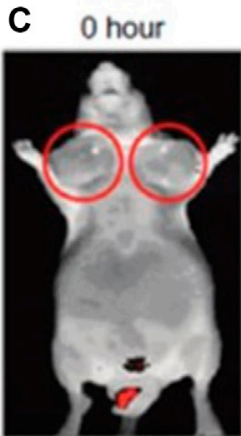

1 day

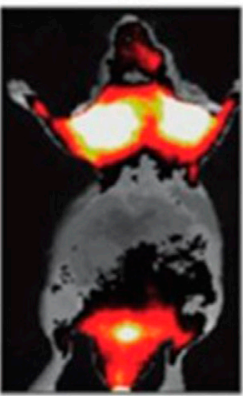

6 hours

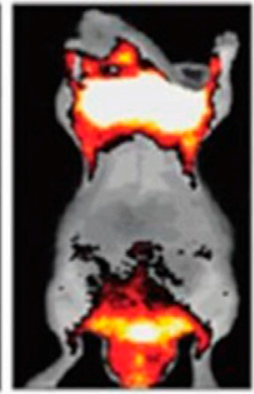

2 days

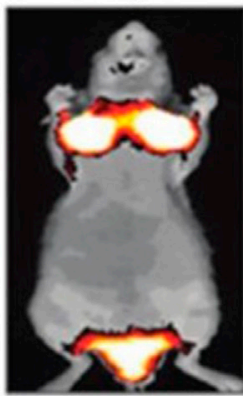

12 hours

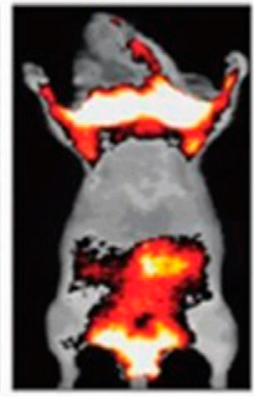

3 days

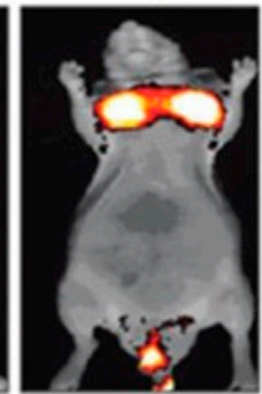

FIGURE 1 | (A-C) In vivo magnetic resonance (MR)-near-infrared fluorescent (NIRF) dual-modality imaging of SCC7-bearing mice. Cy5.5-chitosan nanoparticleGd(III) nanoparticles were injected into the SCC7-bearing mice, and the mice were visualized by using MR and NIRF imaging. Red circles indicate tumor sites. (A) In vivo MR imaging showed T1-positive contrast effects $1 \mathrm{~h}$ after injection at the tumor sites. (B) Both NIRF and T1-weighted MR images were simultaneously observed after 1 day post injection of Cy5.5-CNP-Gd(III). In vivo NIRF imaging showed brighter NIRF intensity at the tumor site. In vivo MR imaging $24 \mathrm{~h}$ after injection showed bright contrast effects at the tumor site. (C) In vivo NIRF imaging showed the accumulated Cy5.5-CNP-Gd(III) nanoparticles over time at the tumor sites. Reprinted (adapted) with permission from [44], Nam et al., 2010). Copyright (2021) American Chemical Society.

imaging agents directed to tumors. They can provide simultaneously diagnostic information and targeted drug delivery by photon-based stimulus through fluorescence [56], photodynamic [57, 58] and photothermal treatment [57].

In modern anti-cancer modalities, the majority approaches the delivering of new drugs which are behaving as a "Trojan horse", by introducing the active, cytotoxic compound in a nanoparticle and "decorating" its surface with a ligand that trigger the cancer cell into taking it up. Imaging can be used to trace the delivery of the drug inside the body and simultaneously to activate the release of the drug by an external stimulus such as laser light $[59,60]$. Functionalized nanoparticles can act both as contrast agents and photosensitizers for photothermal (PT) [61] or photodynamic treatment (PDT) [62]. Among the large variety of NPs, metal NPs (AuNPs) are in the cutting edge of the nanomedicine due to their unique physical, optical and electronic properties $[63,64]$. When metal NPs excited by visible or infrared monochromatic light with laser wavelength corresponding to their Surface Plasmon Resonance (SPR), the conduction electrons of the metal can be subjected to coherently oscillation and convert the electromagnetic energy into heat providing targeted tumour disruption via hyperthermic damage [65]. Photodynamic therapy has come again to the forefront due to the new class of photosensitizers (PS) which enhance PTD efficiency. The encapsulation of PS such as verteporfin or methylene blue into nanocarriers seems to overcome some of the barriers of the PS i.e., poor selectivity to the target tissues, the low extinction coefficients, their lipophilicity, the photobleaching of the PS etc. The synergia of nanomedicine with biophotonic techniques could lead to a localized "surgery" causing tumor disruption or removal without invasiveness [62].

Nano-image guided surgery plays an emerging role in the field of personalized tumour surgery $[65,66]$. Fluorescence-imaging guided surgery can be used for sentinel lymph node mapping or to distinguish the margins of a tumor in microscopic scale and in real time. Using fluorescence imaging in the near-infrared (NIR) window $(700-1,300 \mathrm{~nm})$ is superior to visible light due to high penetration depth, negligible tissue autofluorescence, low scattering offering higher sensitivity and better signal-to-noise ration. Nanoparticles like quantum dots, liposomes or supermagnetic NPs can be conjugated with NIR fluorescence dyes acting as targeted optical imaging probes to offer high selectivity and specificity [67]. Recently, Upconverting Nanoparticles (UCNPs) have been introduced in Biophotonics and nanophotonics as very promising theranostic intratissue probes in biological tissues $[68,69]$. Their unique property to convert near-infrared (NIR) light into visible or ultraviolet light via photon upconversion mechanism will permit interventions to deeper tissues pathologies with minimum healthy cells destruction. 


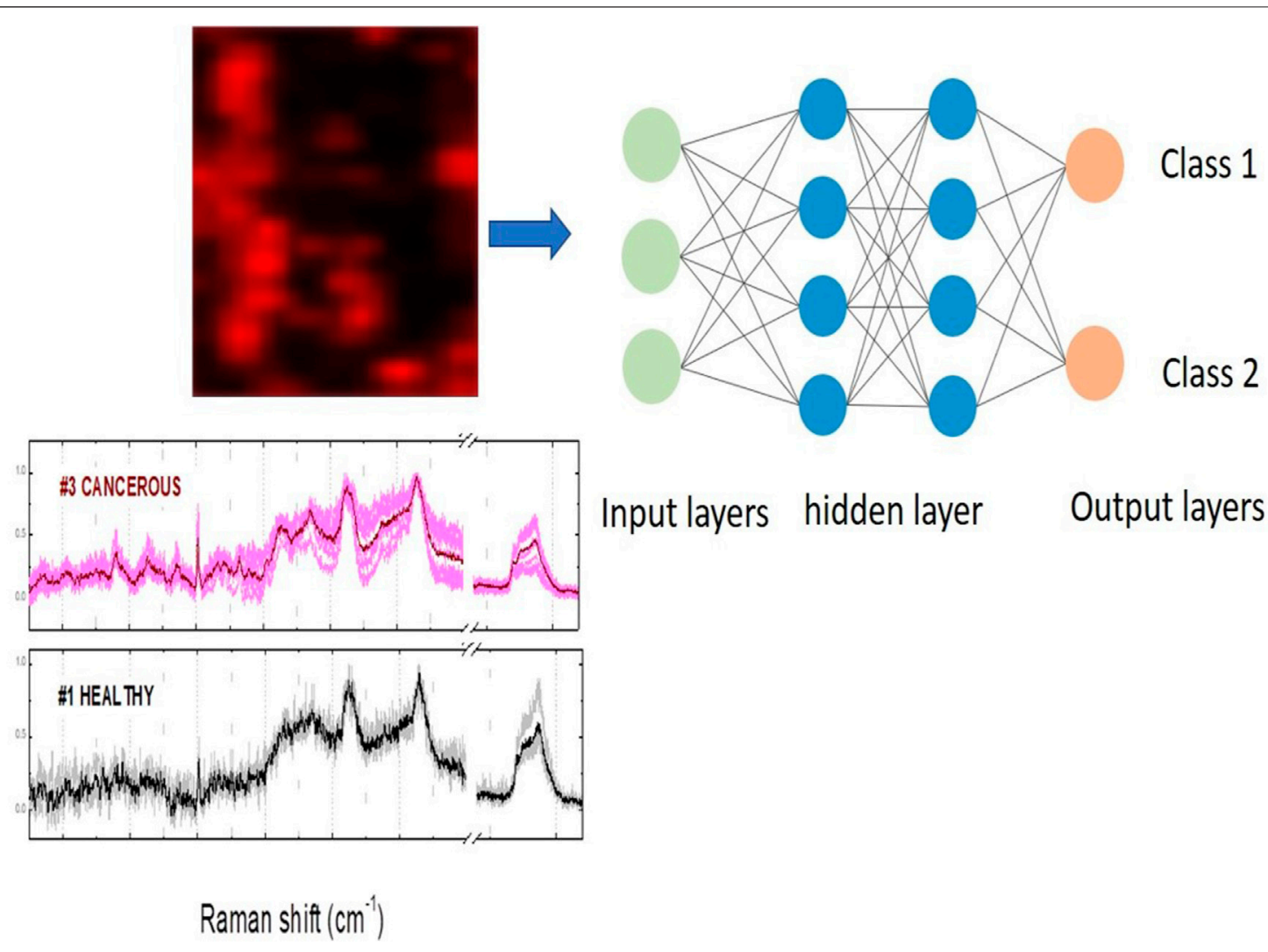

FIGURE 2|A schematic illustration of machine learning methodology applied in Raman spectroscopy. Image features or spectral images are used as inputs to the artificial intelligence algorithm and the class output can be normal or tumour for discrimination between healthy and cancerous tissues.

\section{THE ROLE OF OPTICAL TWEEZERS IN BIOPHOTONICS}

Over 30 years of exploration after the first report of damage-free optical trapping of virus and bacteria by Ashkin [70], optical tweezers have found innumerable applications in cell biology and living systems studies [71]. The optical trapping technique uses one or more laser beams to selectively manipulate position, motion, and dynamics of micro- and nanostructures. This phenomenon is based on the optical forces of the order of few piconewtons exerted as the electromagnetic radiation (photons) changes its momentum when it interacts with matter. For the theoretical and experimental study of the optical forces, two models have been developed, considering the particle diameter (d) compared to the wavelength $(\lambda)$ of the incident light: the geometric model that refers to the case where the dimensions of the particle are much larger than the wavelength (Mie particle, $d>>\lambda)[72,73]$ and the electromagnetic model for particles of dimensions much smaller than the wavelength (Rayleigh particle, $\mathrm{d}<<\lambda$ ) [74, 75]. In case of $\mathrm{d} \sim \lambda$, generalized electromagnetic theories have been applied such as the generalized Lorentz-Mie theory, which describes the scattering of a flat electromagnetic wave by a sphere of any size, in the case of Gaussian bonds [76]. The optical force exerted on a cell depends on the particle's shape, size, its surface and the cytoplasmic refractive index. Therefore, the biochemical changes that happens in the cell cytoplasm or membrane are reflected to its behavior under the optical trap [77].

Optical tweezers are used extensively for studying living cells, e.g., for hemorheology studies, blood microcirculation and biomechanical properties [77, 78]Click or tap here to enter text. They can be used as passive "force clamps" to induce and study elastic deformations in individual cells [79]Click or tap here to enter text. They are a tool for calculating the stiffness and torsion rate by measuring sub-micrometric cell deformations, which are caused by optical forces. Researchers used optical tweezers to elongate human erythrocytes through the dual optical trapping of silicone spheres attached to the cell membrane or by line optical tweezers (Figure 3) and to determine their degree of torsion. The shear modulus was calculated by measuring the cell membrane deformation in function with the optical forces exerted to the membrane via small optically trapped silica beads [80]Click or tap here to enter text. The bending modulus of the membrane was estimated by measuring erythrocyte's folding time in function with laser power under the effect of line optical tweezers [81]Click or tap here to enter text. Optical tweezers have been used to induce rotation and folding of erythrocytes to study their elastic properties and diagnose malaria in them $[78,82]$. Click or tap here to enter text. Zhao et al [83]Click or tap here to enter text. proposed and implemented an optical shield scheme, based in far-field Bessel 

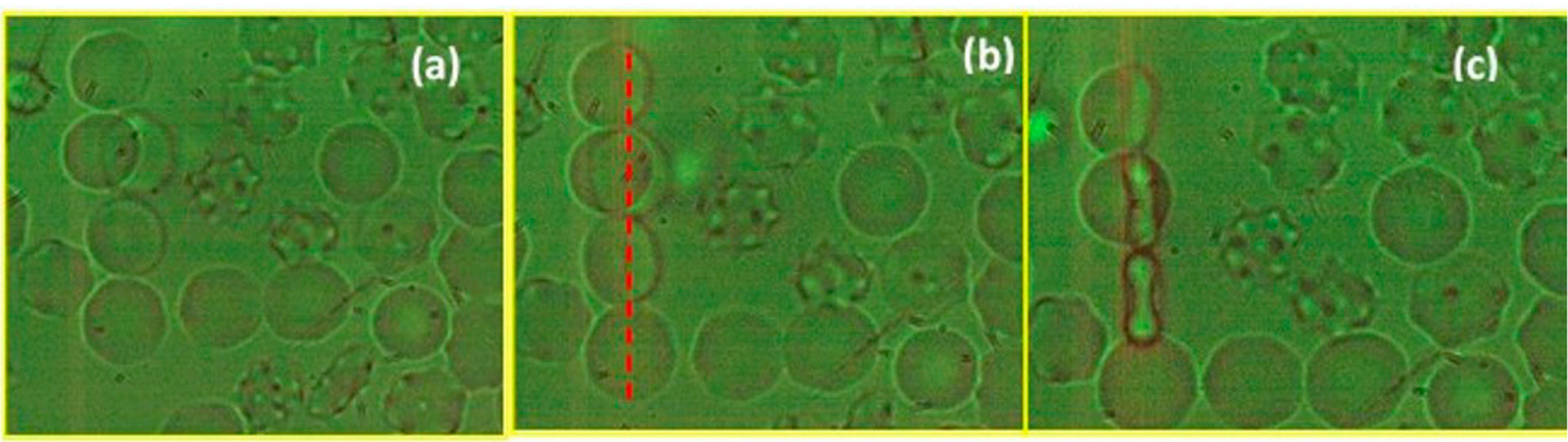

FIGURE 3 | (A) Three red blood cells under a line optical tweezers. The RBCs are trapped simultaneously, (B) are folded gradually and (C) orient its long axis in the direction of the electric field of incident beam. Dotted red line is the direction of the line optical trap. Optical tweezers act as a tool for the evaluation of erythrocyte's deformability which is an important biomarker for circulation efficiency.

beam, for manipulating individual cells in a crowded environment (e.g., single blood cell, individual lymphocytes from an inguinal lymph node). Holographic optical tweezers have been developed by using spatial light modulators to trap and move many cells simultaneously [84, 85]. This offers the opportunity to create arrays of living cells into gel matrix or microfluidic networks and potentially to create artificial tissues [86]. Line optical tweezers created with holographic optical trapping technique have been used for measuring the effective interaction potential for pairs of colloidal particles [87].

In addition to other biomedical areas, there have been great advances in optical trapping and its combination with other Biophotonics tools in neuroscience research, for studying the physical properties and intrinsic forces of neurons, their communication modalities, as well as some of the fundamental neuronal growth and dynamics function [88]. Optical tweezers can be combined with Raman spectroscopy to characterize and monitor the physical and chemical properties of cells. For example, Laser tweezers Raman spectroscopy was used to monitor the changes in the oxygenation state of human red blood cells while they were stretched by the optical forces [89, 90]Click or tap here to enter text.

So far, no clinical application in human has been implemented by using optical tweezers. Infrared optical tweezers have been used to trap and manipulate erythrocytes in the blood capillaries in the ear of a mouse. Optical tweezers were capable to interfere to the blood stream by trapping erythrocytes or removing a blockage [91]Click or tap here to enter text. A very challenging also demand is the miniaturization of the biophotonic set-ups opening new, fascinating possibilities for in vitro single cell experiments like flow cytometry, laser induced fluorescence and for in vivo detection of diseases. Optical tweezers can be coupled with nanophotonic biosensor devices based on integrated fiber optics and microfluidics devices for the implementation of lab-on-a-chip platforms. They can probe complex biophysical and biomechanical processes governing cell-cell interactions, cell-surface interactions, cell sorting and drug delivery/testing [92-94]Click or tap here to enter text. Figure 4 illustrates a simple implementation of an advanced optical tweezes system integrating with microfluidics devices to perform single cell manipulation and sorting between target with NPs cancer cells and healthy cells. Moreover, the selective observation of cellsnanoparticles interactions will lead to a better understanding of the interaction mechanisms and to more targeted treatments.

\section{THE APPLICATION OF OPTICAL TWEEZERS IN CANCER FIELD AND FUTURE PERSPECTIVES}

The ability to selectively manipulate single cells can have many advantages in vivo for the diagnosis and treatment of metastatic cancer cells which can travel through the bloodstream and the lymph system [79, 95]Click or tap here to enter text. The understanding the biophysics of individual cell deformation offers the means for new perspectives in cancer prognosis, diagnosis, and treatment. Changes in the ability to deform cell shape [96] combined with changes in cell adhesion affect cell reproduction, cell signal transmission, and cell metastasis potential [97]. Qian Zhao et al. demonstrated the optical manipulation of two lymphocytes under living conditions. The lymphocytes were optically trapped directly as they isolated from a lymph node using a Bessel beam created by an axicon and a lens [83]. Trapping and manipulation of single cells in living environments is expected to help the study of how natural killer cells react to cancer cells or to selectively bring killer cells into contact with other target cells [83].

Researchers have reported the use of optical trapping as a tool to measure the minimum cell-cell adhesion time as a line cell is trapped and brought into proximity to another [95]. They observed that the average minimum adhesion time increases significancy in neural tumor cells compared to healthy cells. Moreover, they induced chemically differentiation in various cell line and tumors and proved that optical tweezers are able to assess the differentiation status of cancerous cells by measuring the minimum cell-cell adhesion time. Discrimination of individual cancer cells have been also reported by using Laser Tweezers Raman Spectroscopy coupling with a microfluidic flow 


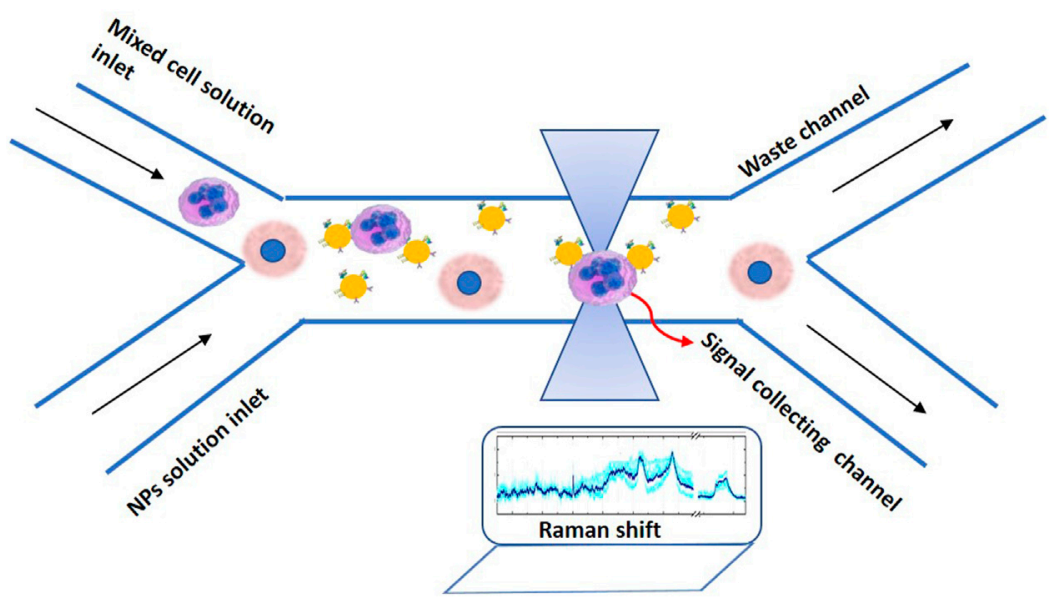

FIGURE 4 | Schematic representation of dual-beam optical tweezers coupled with microfluidics device to manipulate and recognize targeted with functionalized NPs tumour cells from healthy cells. The system can be integrated with other spectroscopic set-ups such as a Raman microscope to collect signals for cell discrimination and characterization.

chamber [98]. Single cells are optically trapped, analyzed and discriminate according to the differences in their spectral fingerprint [98, 99].

Holographic optical tweezers can be used to discriminate normal, cancerous and drug-treated cancerous leucocytes by measuring the trapping forces using escape force method [100]. This method could become equivalent with the conventional methods such as flow cytometry without using fluorescent-based markers. Moreover, holographic optical tweezers can be combined with upconversion luminescence encoding for screening cancer biomarkers [101]. A bead array of carboxyl functionalized polystyrene beads was formed and stay stable with holographic optical tweezers. The beads were labelled with upconverting nanoparticles probes (UCNPs) of two different emission colors for the detection of two liver cancer biomarkers, carcinoembryonic antigen and alpha fetal protein. UCNPs are able to excited from near-infrared (NIR) light region and emitted in the visible region with extremely low background luminescence. This imaging-based stable suspension array offers the detection of dual cancer biomarkers with quite sensitivity and specificity providing a new alternative method for cancer diagnosis [101].

The mechanical properties of cancer cells membranes such as bending modulus [102] and fluidity [103] have been also measured by using optical tweezers and the standard stretching methods with the microbeads attached to the cell membrane [80]. The biomechanical properties of living cells are closely related to the health status and function of human cells $[96,102,104]$. Guo et al. measure with accuracy the bending modulus and the surface membrane tension of breast cancer cells [105]. Moreover, decreased elongation times are recorded when cells were treated with cytochalasin D or the membrane protein caveolin was over-expressed. Xuanling Li et al. showed that the fluidity and the invasiveness in the membrane of small cell lung cancer cell line SHP77 increased significancy after the transfection with small RNA miR-92b-
$3 \mathrm{p}$. This could help to understand better cancer cell metastasis/ invasion [106].

High-precision optical tweezers have been developed for protein folding experiments. Optical tweezers were used to apply mechanical forces and to monitor proteins unfolding [107]. Recent studies correlate the folding and misfolding of human membrane proteins with cancer [108]. The way that proteins fold from linear chains to three-dimensional structures or vice versa is under great interest in biology [109, 110]. Single proteins or protein complex can be tethered between two microbeads by using DNA linkers or antibody linkers. The beads are trapped by using a dual optical tweezers and can be pulled away by alter the distance between the laser traps. Thus, protein unfolding is induced by the mechanical forces and the kinetics of the protein can be monitoring [107, 111] The mechanical forces at which folding transitions take place depend on the pulling speed [111].

During the last few years, optical tweezers were proposed as a tool to probe the Casimir interactions between microspheres inside a liquid medium, erythrocytes and membrane proteins [112-114]. The Casimir effect is a quantum phenomenon arising from quantum fluctuations which can give rise to long-range attractive forces between two uncharged particles [112]. Recent studies demonstrate that the Casimir forces between two particles can be measured by the optical trapping of the particles in suspensions [115]. Physicists manifest that the proteins of cellular membranes can communicate with each other by using Casimir forces. Examples include the force exerted by a single DNA molecule and forces in kinesin or other moto proteins [116]. This finding gives to optical tweezers technique the potential for novel quantitative applications in molecular biology of cancer.

Nowadays, advanced optical tweezers platforms have been built-up to operate at single-molecule level without the need of fluorescence dyes or tethers. Plasmonic tweezers are capable to manipulate with high sensitivity molecules such as proteins, DNA etc. $[117,118]$ attached on metallic nanostructures exploiting the Localized Surface Plasmon Resonance (LSPR) phenomenon 


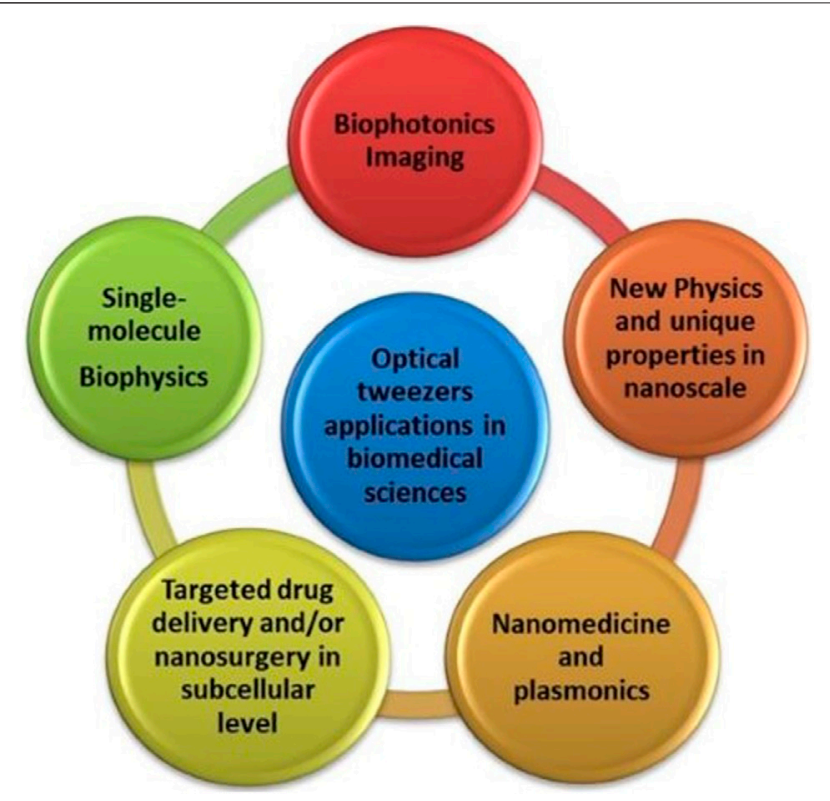

FIGURE 5 | Schematic illustration of some interconnected components of advanced Biophotonics techniques for cellular and molecular manipulation through optical tweezers.

which can exist on a dielectric-metal interface [119]. LSPR is a coherent, collective spatial oscillation of the conduction electrons in a metal nanostructure, which can be directly excited by visible and near infrared light. The SPR occurs when the real part $\varepsilon_{r}(\omega)$ of the complex dielectric constant of the metal and the dielectric constant of the surrounding medium satisfied the relationship: $\varepsilon_{\mathrm{r}}(\omega)=-2 \varepsilon_{\mathrm{m}}$ [120]. This phenomenon enables the incident light to confine into a region smaller than the light wavelength enhancing the oscillating electric field of the light which strengthens the optical forces.

Gordon and his collaborators have developed novel plasmonic configurations which provide stable and flexible traps for biomolecules [121, 122]. Very recently, the smallest virus particle, PhiX174, was optically trapped by using double nanohole apertures in gold nanofilms. The virus was analyzed by using Plasmon Tweezers integrating with Raman Spectroscopy [123]. Plasmonic tweezers have also thermal effects through the resonated oscillations of the conductive electrons of the metallic nanostructures which convert the electromagnetic energy into heat. This might give new perspectives in photothermal cancer therapy for a more targeted treatment. Already, plasmonic photothermal therapy was studied extensively based on various types of metallic nanoparticles as photosensitizers [124, 125]. Moreover, in 2021, Shen et al. reported that UCNPs from highly

\section{REFERENCES}

1. Popp J, Tuchin VV, Chiou A, Heinemann SH. Handbook of Biophotonics: Vol. 1: Basics and Techniques, Volume 1: Basics and Techniques. 1st ed. (2008). doping lanthanide ions in NaYF4 nanocrystals can be optically manipulated and demonstrate much higher optical trap stiffness compared to gold nanoparticles [126]. The photoluminescence of UPCNPs could provide new fascinating theranostic interventions by single-cell manipulation and sensing. Figure $\mathbf{5}$ illustrates all the interconnected multidisciplinary fields of the advanced Biophotonics techniques for cellular and molecular manipulation through optical tweezers.

\section{CONCLUSION}

Biophotonic techniques have proved to be a powerful tool in the field of cancer theranostics. Among them, optical tweezers can spur new approaches to cancer treatment and to the understanding of cancer mechanisms at cellular and subcellular level. Nowadays, integrated systems based to optical tweezers are capable to manipulate to each other cells, biomolecules and nanocarriers with high sensitivity and selectivity providing information about their biomechanical properties (e.g., membrane fluidity, elasticity etc), their biochemical and biophysical properties (e.g., spectral fingerprints) and tracking their interactions (e.g., adhesion time, adhesion forces etc). Overall, the unique capabilities of optical tweezers in combination with the development of advanced miniaturized devices and artificial intelligence methodologies will be a boon for the cancer confrontation.

\section{AUTHOR CONTRIBUTIONS}

Conceptualization, writing-original draft preparation ES.

\section{FUNDING}

This research has been co-financed by the European Regional Development Fund of the European Union and Greek national funds through the Operational Program Competitiveness, Entrepreneurship and Innovation, under the call RESEARCH-CREATE-INNOVATE (project code: T2EDK-01223). Development of advanced portable biophotonic system for the personalized spectroscopic discrimination of cancer margins/tissues, Acronym: BIOPHASMA).

\section{ACKNOWLEDGMENTS}

The authors would like to thank Prof. M. Labropoulou for her constructive comments on the manuscript.

2. Serafetinides AA, Makropoulou M, Drakaki E. Biophotonics Diagnosis Modeling Tissue Pathologies Proc SPIE 15th Int Sch Quan Electron Laser Phys Appl (2008) 7027:702715. doi:10.1117/12.822517

3. Brownstein M, Hoffman RA, Levenson R, Milner TE, Dowell ML, Williams PA, et al. Biophotonic Tools in Cell and Tissue Diagnostics. J Res Natl Inst Stand Technol (2007) 112:139. doi:10.6028/jres.112.011 
4. Tsia K. Understanding Biophotonics: Fundamentals, Advances, and Applications. 1st ed. (2015).

5. Pradad PN. Fundamentals of Nanobiophotonics. In: Photon-based Nanoscience and Nanobiotechnology, Vol. 239. , DordrechtNetherlands: Springer (2006). p. 55-65. doi:10.1007/978-1-4020-5523-2

6. Prasad PN. Nanobiophotonics and Nanoclinics for Multimodal Imaging and Targeted Therapy. In Frontiers in Optics 2007/Laser Science XXIII/Organic Materials and Devices for Displays and Energy Conversion, OSA. Washington, DC (2007).

7. Chacko JV. Nanobiophotonics and Fluorescence Nanoscopy in 2020. NanoOptics, Elsevier (2020). p. 113-62. doi:10.1016/B978-0-12-818392-2.00006-8

8. Soloperto A, Palazzolo G, Tsushima H, Chieregatti E, Vassalli M, Difato F. Laser Nano-Neurosurgery from Gentle Manipulation to Nano-Incision of Neuronal Cells and Scaffolds: An Advanced Neurotechnology Tool. Front Neurosci (2016) 10:101. doi:10.3389/fnins.2016.00101

9. Saquilabon Cruz GM, Kong X, Silva BA, Khatibzadeh N, Thai R, Berns MW. Femtosecond Near-Infrared Laser Microirradiation Reveals a Crucial Role for PARP Signaling on Factor Assemblies at DNA Damage Sites. Nucleic Acids Res (2016) 44:e27. doi:10.1093/nar/gkv976

10. Difato F, Dal Maschio M, Marconi E, Ronzitti G, Maccione A, Fellin T, et al. Combined Optical Tweezers and Laser Dissector for Controlled Ablation of Functional Connections in Neural Networks. J Biomed Opt (2011) 16:051306. doi:10.1117/1.3560268

11. Habibey R, Golabchi A, Latifi S, Difato F, A Microchannel Device Tailored to Laser Axotomy and Long-Term Microelectrode Array Electrophysiology of Functional Regeneration. Lab Chip (2015) 15:4578-90. doi:10.1039/C5LC01027F

12. Seeger S, Monajembashi S, Hutter K-J, Futterman G, Wolfrum J. Application of Laser Optical Tweezers in Immunology and Molecular Genetics. Cytometry (1991) 12:497-504. doi:10.1002/cyto.990120606

13. Byun H, Hillman TR, Higgins JM, Diez-Silva M, Peng Z, Dao M, et al. Optical Measurement of Biomechanical Properties of Individual Erythrocytes from a Sickle Cell Patient. Acta Biomater (2012) 8:4130-8. doi:10.1016/j.actbio.2012. 07.011

14. Choudhary D, Mossa A, Jadhav M. Bio-Molecular Applications of Recent Developments in Optical Tweezers. Biomolecules (2019) 9:23. doi:10.3390/ biom9010023

15. Coirault C, Pourny J-C, Lambert F. Les pinces optiques en biologie et en médecine. Med Sci (Paris) (2003) 19:364-7. doi:10.1051/medsci/2003193364

16. Polimeno P, Magazzù A, Iatì MA, Patti F, Saija R, Esposti Boschi CD, et al. Optical Tweezers and Their Applications. J Quantitative Spectrosc Radiative Transfer (2018) 218:131-50. doi:10.1016/j.jqsrt.2018.07.013

17. Tam JM, Castro CE, Heath RJ, Cardenas ML, Xavier RJ, Lang MJ, et al. Control and Manipulation of Pathogens with an Optical Trap for Live Cell Imaging of Intercellular Interactions. PLoS ONE (2010) 5:e15215. doi:10. 1371/journal.pone.0015215

18. Kumar R, Gulia K, Chaudhary M, Shah MA. SARS-CoV-2, Influenza Virus and Nanoscale Particles Trapping, Tracking and Tackling Using Nanoaperture Optical Tweezers: A Recent Advances Review. J Mat Nanosci (2020) 7:79-92.

19. Serafetinides AA, Makropoulou M, Kotsifaki DG, Tsigaridas G. Biophotonics Imaging Cell Manipulation: Quo Vadis?, T Dreischuh, S Gateva, A Daskalova, A Serafetinides Editors (2017) 1022613. doi:10.1117/12.2263036

20. Holzner G, Mateescu B, van Leeuwen D, Cereghetti G, Dechant R. Highthroughput Multiparametric Imaging Flow Cytometry: toward DiffractionLimited Sub-cellular Detection and Monitoring of Sub-cellular Processes. Cel Rep (2021) 34:108824. doi:10.1016/j.celrep.2021.108824,

21. Liu L, Yang Q, Zhang M, Wu Z. Fluorescence Lifetime Imaging Microscopy and its Applications in Skin Cancer Diagnosis. J Innov Opt Health Sci (2019) 12:1930004. doi:10.1142/S1793545819300040

22. Rashid R, Gaglia G, Chen Y-A, Lin J-R, Du Z, Maliga Z, et al. Highly Multiplexed Immunofluorescence Images and Single-Cell Data of Immune Markers in Tonsil and Lung Cancer. Sci Data (2019) 6:323. doi:10.1038/ s41597-019-0332-y

23. Țălu SD. Optical Coherence Tomography in the Diagnosis and Monitoring of Retinal Diseases. ISRN Biomed Imaging (2013) 2013:1-13. doi:10.1155/2013/ 910641

24. Freeman EE, Semeere A, Osman H, Peterson G, Rajadhyaksha M, González S, et al. Smartphone Confocal Microscopy for Imaging Cellular Structures in
Human Skin In Vivo. Biomed Opt Express (2018) 9:1906. doi:10.1364/BOE.9. 001906

25. He D, Wang H, Ho S-L, Chan H-N, Hai L, He X, et al. Total Internal ReflectionBased Single-Vesicle In Situ Quantitative and Stoichiometric Analysis of Tumor-Derived Exosomal microRNAs for Diagnosis and Treatment Monitoring. Theranostics (2019) 9:4494-507. doi:10.7150/thno.33683

26. Benninger RK, Piston DW. Two-Photon Excitation Microscopy for the Study of Living Cells and Tissues. Curr Protoc Cel Biol (2013) Chapter 4:Unit. doi:10.1002/0471143030.cb0411s59

27. Sekar RB, Periasamy A. Fluorescence Resonance Energy Transfer (FRET) Microscopy Imaging of Live Cell Protein Localizations. J Cel Biol. (2003) 160(5):629-33. doi:10.1083/jcb.200210140

28. Balagopalan L, Sherman E, Barr VA. Imaging Techniques for Assaying Lymphocyte Activation in Action. Nat Rev Immunol (2011) 11:21-33. doi:10.1038/nri2903

29. Gourley PL, Hendricks JK, McDonald AE, Copeland RG, Barrett KE, Gourley CR, et al. Mitochondrial Correlation Microscopy and Nanolaser Spectroscopy - New Tools for Biophotonic Detection of Cancer in Single Cells. Technol Cancer Res Treat (2005) 4:585-92. doi:10.1177/153303460500400602

30. Leary J, Key J. Nanoparticles for Multimodal In Vivo Imaging in Nanomedicine. Ijn (2014) 2014:711-26. doi:10.2147/IJN.S53717

31. Zhu B, Sevick-Muraca EM. A Review of Performance of Near-Infrared Fluorescence Imaging Devices Used in Clinical Studies. Bjr (2015) 88: 20140547. doi:10.1259/bjr.20140547

32. Feng J, Chang D, Wang Z, Shen B, Yang J, Jiang Y, et al. A FITC-Doped Silica Coated Gold Nanocomposite for Both In Vivo X-ray CT and Fluorescence Dual Modal Imaging. RSC Adv (2014) 4:51950-9. doi:10.1039/C4RA09392E

33. Xie J, Chen K, Huang J, Lee S, Wang J, Gao J, et al. PET/NIRF/MRI Triple Functional Iron Oxide Nanoparticles. Biomaterials (2010) 31:3016-22. doi:10.1016/j.biomaterials.2010.01.010

34. Zhang J, Li C, Zhang X, Huo S, Jin S, An F-F, et al. In Vivo tumor-targeted Dual-Modal Fluorescence/CT Imaging Using a Nanoprobe Co-loaded with an Aggregation-Induced Emission Dye and Gold Nanoparticles. Biomaterials (2015) 42:103-11. doi:10.1016/j.biomaterials.2014.11.053

35. Nam T, Park S, Lee S-Y, Park K, Choi K, Song IC, et al. Tumor Targeting Chitosan Nanoparticles for Dual-Modality Optical/MR Cancer Imaging. Bioconjug Chem. (2010) 21:578-82. doi:10.1021/bc900408z

36. Cai W, Chen K, Li Z-B, Gambhir SS, Chen X. Dual-Function Probe for PET and Near-Infrared Fluorescence Imaging of Tumor Vasculature. J Nucl Med (2007) 48:1862-70. doi:10.2967/jnumed.107.043216

37. Pradhan P, Guo S, Ryabchykov O, Popp J, Bocklitz TW. Deep Learning a Boon for Biophotonics. J Biophotonics (2020) 13:e201960186. doi:10.1002/ jbio.201960186

38. Fan H, Zhang F, Xi L, Li Z, Liu G, Xu Y. LeukocyteMask: An Automated Localization and Segmentation Method for Leukocyte in Blood Smear Images Using Deep Neural Networks. J Biophotonics (2019) 12:e201800488. doi:10. 1002/jbio.201800488

39. Wang H, Rivenson Y, Jin Y, Wei Z, Gao R, Günaydın H, et al. Deep Learning Enables Cross-Modality Super-resolution in Fluorescence Microscopy. Nat Methods (2019) 16:103-10. doi:10.1038/s41592-018-0239-0

40. Liu J, Osadchy M, Ashton L, Foster M, Solomon CJ, Gibson SJ. Deep Convolutional Neural Networks for Raman Spectrum Recognition: a Unified Solution. Analyst (2017) 142:4067-74. doi:10.1039/C7AN01371J

41. Lussier F, Missirlis D, Spatz JP, Masson JF. Machine-Learning-Driven Surface-Enhanced Raman Scattering Optophysiology Reveals Multiplexed Metabolite Gradients Near Cells. ACS Nano (2019) 13:1403-11. doi:10.1021/ acsnano.8b07024

42. Ou L, Chen Y, Su Y, Huang Y, Chen R, Lei J. Application of Silver Nanoparticle-Based SERS Spectroscopy for DNA Analysis in Radiated Nasopharyngeal Carcinoma Cells. J Raman Spectrosc (2013) 44:680-5. doi:10.1002/jrs.4269

43. Kim W, Lee SH, Kim SH, Lee J-C, Moon SW, Yu JS, et al. Highly Reproducible Au-Decorated $\mathrm{ZnO}$ Nanorod Array on a Graphite Sensor for Classification of Human Aqueous Humors. ACS Appl Mater Inter (2017) 9:5891-9. doi:10.1021/acsami.6b16130

44. Nikitaki Z, Hellweg CE, Georgakilas AG, Ravanat JL. Stress-induced DNA Damage Biomarkers: Applications and Limitations. Front Chem (2015) 3:35. doi:10.3389/fchem.2015.00035 
45. Heylmann D, Rödel F, Kindler T, Kaina B. Radiation Sensitivity of Human and Murine Peripheral Blood Lymphocytes, Stem and Progenitor Cells. Biochim Biophys Acta (Bba) - Rev Cancer (2014) 1846:121-9. doi:10.1016/ j.bbcan.2014.04.009

46. Horn S, Barnard S, Rothkamm K. Gamma-H2AX-Based Dose Estimation for Whole and Partial Body Radiation Exposure. PLoS ONE (2011) 6:e25113. doi:10.1371/journal.pone.0025113

47. Chen F, Ehlerding EB, Cai W. Theranostic Nanoparticles. J Nucl Med (2014) 55:1919-22. doi:10.2967/jnumed.114.146019

48. Madamsetty VS, Mukherjee A, Mukherjee S. Recent Trends of the BioInspired Nanoparticles in Cancer Theranostics. Front Pharmacol (2019) 10: 1264. doi:10.3389/fphar.2019.01264

49. Zavaleta C, Ho D, Chung EJ. Theranostic Nanoparticles for Tracking and Monitoring Disease State. SLAS TECHNOLOGY: Translating Life Sci Innovation (2018) 23:281-93. doi:10.1177/2472630317738699

50. Menon JU, Jadeja P, Tambe P, Vu K, Yuan B, Nguyen KT. Nanomaterials for Photo-Based Diagnostic and Therapeutic Applications. Theranostics (2013) 3:152-66. doi:10.7150/thno.5327

51. Jaque D, Martínez Maestro L, del Rosal B, Haro-Gonzalez P, Benayas A, Plaza JL, et al. Nanoparticles for Photothermal Therapies. Nanoscale (2014) 6: 9494-530. doi:10.1039/C4NR00708E

52. Yang L, Sajja HK, Cao Z, Qian W, Bender L, Marcus AI, et al. uPAR-Targeted Optical Imaging Contrasts as Theranostic Agents for Tumor Margin Detection. Theranostics (2014) 4:106-18. doi:10.7150/thno.7409

53. Harlaar NJ, Kelder W, Sarantopoulos A, Bart J, Themelis G, van Dam GM, et al. Real-time Near Infrared Fluorescence (NIRF) Intra-operative Imaging in Ovarian Cancer Using an av $\beta 3$-integrin Targeted Agent. Gynecol Oncol (2013) 128:590-5. doi:10.1016/j.ygyno.2012.12.011

54. Yasun E. Theranostic Cancer Applications Utilized by Nanoparticles Offering Multimodal Systems and Future Insights. SN Appl Sci (2020) 2:1552. doi:10. 1007/s42452-020-03397-4

55. Gao X, Wang Q, Cheng C, Lin S, Lin T, Liu C, et al. The Application of Prussian Blue Nanoparticles in Tumor Diagnosis and Treatment. Sensors (2020) 20:6905. doi:10.3390/s20236905

56. Patsenker L, Gellerman G. Fluorescent Reporters for Drug Delivery Monitoring. Isr J Chem (2020) 60:504-18. doi:10.1002/ijch.201900137

57. Shibu ES, Hamada M, Murase N, Biju V. Nanomaterials Formulations for Photothermal and Photodynamic Therapy of Cancer. J Photochem Photobiol C: Photochem Rev (2013) 15:53-72. doi:10.1016/j.jphotochemrev.2012.09.004

58. Yang Z, Sun Z, Ren Y, Chen X, Zhang W, Zhu X, et al. Advances in Nanomaterials for Use in Photothermal and Photodynamic Therapeutics (Review). Mol Med Rep (2019) 20:5-15. doi:10.3892/mmr.2019.10218

59. Sarkar SK, Khater Y, Kulkarni A, Sengupta S. Feedback-mediated Cancer Therapy: a FRET-Based Nanoreporter Approach. H Mohseni, M.H Agahi, M Razeghi (2014) 916607. doi:10.1117/12.2061379

60. Luo Y, Huang L, Yang Y, Zhuang X, Hu S, Ju H, et al. A Programmed Nanoparticle with Self-Adapting for Accurate Cancer Cell Eradication and Therapeutic Self-Reporting. Theranostics (2017) 7:1245-56. doi:10.7150/ thno.18187

61. Kim M, Lee JH, Nam JM. Plasmonic Photothermal Nanoparticles for Biomedical Applications. Adv Sci (2019) 6:1900471. doi:10.1002/advs. 201900471

62. Yi G, Hong SH, Son J, Yoo J, Park C, Choi Y, et al. Recent Advances in Nanoparticle Carriers for Photodynamic Therapy. Quant Imaging Med Surg (2018) 8:433-43. doi:10.21037/qims.2018.05.04

63. Rengan AK, Kundu G, Banerjee RR, Srivastava R. Gold Nanocages as Effective Photothermal Transducers in Killing Highly Tumorigenic Cancer Cells. Part Part Syst Charact (2014) 31:398-405. doi:10.1002/ppsc.201300173

64. Huang X, El-Sayed IH, Qian W, El-SayedCancer Cell Imaging MA, Photothermal. Cancer Cell Imaging and Photothermal Therapy in the Near-Infrared Region by Using Gold Nanorods. J Am Chem Soc (2006) 128:2115-20. doi:10.1021/ja057254a

65. Khlebtsov NG, Dykman LA. Optical Properties and Biomedical Applications of Plasmonic Nanoparticles. J Quantitative Spectrosc Radiative Transfer (2010) 111:1-35. doi:10.1016/j.jqsrt.2009.07.012

66. Wojtynek NE, Mohs AM. Image-guided Tumor Surgery: The Emerging Role of Nanotechnology. Wiley Interdiscip Rev Nanomed Nanobiotechnol (2020) 12:e1624. doi:10.1002/wnan.1624
67. An H-W, Hou D, Zheng R, Wang M-D, Zeng X-Z, Xiao W-Y, et al. A NearInfrared Peptide Probe with Tumor-specific Excretion-Retarded Effect for Image-Guided Surgery of Renal Cell Carcinoma. ACS Nano (2020) 14: 927-36. doi:10.1021/acsnano.9b08209

68. Wang C, Cheng L, Liu Z. Upconversion Nanoparticles for Photodynamic Therapy and Other Cancer Therapeutics. Theranostics (2013) 3:317-30. doi: $10.7150 /$ thno. 5284

69. Qiu H, Tan M, Ohulchanskyy T, Lovell J, Chen G. Recent Progress in Upconversion Photodynamic Therapy. Nanomaterials (2018) 8:344. doi:10. 3390/nano8050344

70. Ashkin A, Dziedzic JM. Optical Trapping and Manipulation of Viruses and Bacteria. Science (1987) 235:1517-20. doi:10.1126/science.3547653

71. Eriksson M, Leitz G, Fällman E, Axner O, Ryan JC, Nakamura MC, et al. Inhibitory Receptors Alter Natural Killer Cell Interactions with Target Cells yet Allow Simultaneous Killing of Susceptible Targets. J Exp Med (1999) 190: 1005-12. doi:10.1084/jem.190.7.1005

72. Roosen G, Imbert C. Optical Levitation by Means of 2 Horizontal Laser Beams - Theoretical and Experimental Study. Phys Let (1976) 59.

73. Hulst HC. Light Scattering by Small Particles. New York (: Dover Press (1981). p. 114-27.

74. Wright WH, Sonek GJ, Berns MW. Parametric Study of the Forces on Microspheres Held by Optical Tweezers. Appl Opt (1994) 33:1735-48. doi:10. 1364/ao.33.001735

75. Poynting JH. 1884XV. On the Transfer of Energy in the Electromagnetic Field. Phil Trans R Soc (1884) 175:343-61. doi:10.1098/rstl.1884.0016

76. Gouesbet G, Maheu B, Gréhan G. Light Scattering from a Sphere Arbitrarily Located in a Gaussian Beam, Using a Bromwhich Formulation. J Opt Soc Am A (1998) 5:1427-43.

77. Spyratou E, Makropoulou M, Mourelatou EA, Demetzos C. Biophotonic Techniques for Manipulation and Characterization of Drug Delivery Nanosystems in Cancer Therapy. Cancer Lett (2012) 327:111-22. doi:10. 1016/j.canlet.2011.12.039

78. Avsievich T, Zhu R, Popov A, Bykov A, Meglinski I. The Advancement of Blood Cell Research by Optical Tweezers. Rev Phys (2020) 5:100043. doi:10. 1016/j.revip.2020.100043

79. Guck J, Schinkinger S, Lincoln B, Wottawah F, Ebert S, Romeyke M, et al. Optical Deformability as an Inherent Cell Marker for Testing Malignant Transformation and Metastatic Competence. Biophysical J (2005) 88: 3689-98. doi:10.1529/biophysj.104.045476

80. Hénon S, Lenormand G, Richert A, Gallet F. A New Determination of the Shear Modulus of the Human Erythrocyte Membrane Using Optical Tweezers. Biophysical J (1999) 76:1145-51. doi:10.1016/S0006-3495(99)77279-6

81. Spyratou E, Makropoulou M, Serafetinides AA. Red Blood Cell Micromanipulation with Elliptical Laser Beam Profile Optical Tweezers in Different Osmolarity Conditions. Proc SPIE Med Laser Appl Laser-Tissue Interactions $V$ (2011) 8092:80920T. doi:10.1117/12.88966110.1364/ecbo. 2011.80920t

82. Mohanty K, Mohanty S, Monajembashi S, Greulich KO. Orientation of Erythrocytes in Optical Trap Revealed by Confocal Fluorescence Microscopy. J Biomed Opt (2007) 12:060506. doi:10.1117/1.2822365

83. Zhao Q, Wang H-W, Yu P-P, Zhang S-H, Zhou J-H, Li Y-M, et al. Trapping and Manipulation of Single Cells in Crowded Environments. Front Bioeng Biotechnol (2020) 8:422. doi:10.3389/fbioe.2020.00422

84. Padgett M, Di Leonardo R. Holographic Optical Tweezers and Their Relevance to Lab on Chip Devices. Lab Chip (2011) 11:1196-205. doi:10. 1039/c0lc00526f

85. Woerdemann M, Alpmann C, Esseling M, Denz C. Advanced Optical Trapping by Complex Beam Shaping. Laser Photon Rev (2013) 7:839-54. doi:10.1002/lpor.201200058

86. Mirsaidov U, Scrimgeour J, Timp W, Beck K, Mir M, Matsudaira P, et al. Live Cell Lithography: Using Optical Tweezers to Create Synthetic Tissue. Lab Chip (2009) 8:2174-81. doi:10.1039/b807987k

87. Polin M, Roichman Y, Grier DG. Autocalibrated Colloidal Interaction Measurements with Extended Optical Traps. Phys Rev E Stat Nonlin Soft Matter Phys (2008) 77:051401. doi:10.1103/PhysRevE.77.051401

88. Lenton ICD, Scott EK, Rubinsztein-Dunlop H, Favre-Bulle IA. Optical Tweezers Exploring Neuroscience. Front Bioeng Biotechnol (2020) 8: 602797. doi:10.3389/fbioe.2020.602797 
89. Liu R, Mao Z, Matthews DL, Li C-S, Chan JW, Satake N. Novel Single-Cell Functional Analysis of Red Blood Cells Using Laser Tweezers Raman Spectroscopy: Application for Sickle Cell Disease. Exp Hematol (2013) 41: 656-61. doi:10.1016/j.exphem.2013.02.012

90. Rao S, Bálint Š, Cossins B, Guallar V, Petrov D. Raman Study of Mechanically Induced Oxygenation State Transition of Red Blood Cells Using Optical Tweezers. Biophysical J (2009) 96:209-16. doi:10.1529/biophysj.108.139097

91. Zhong M-C, Wei X-B, Zhou J-H, Wang Z-Q, Li Y-M. Trapping Red Blood Cells in Living Animals Using Optical Tweezers. Nat Commun (2013) 4:1768. doi:10.1038/ncomms2786

92. Wu J, Gu M. Microfluidic Sensing: State of the Art Fabrication and Detection Techniques. J Biomed Opt (2011) 16:080901. doi:10.1117/1.3607430

93. Vanapalli SA, Duits MH, Mugele F. Microfluidics as a Functional Tool for Cell Mechanics. Biomicrofluidics (2009) 3:12006. doi:10.1063/1.3067820

94. Monat C, Domachuk P, Grillet C, Collins M, Eggleton BJ, Cronin-Golomb M, et al. Optofluidics: a Novel Generation of Reconfigurable and Adaptive Compact Architectures. Microfluid Nanofluid (2008) 4:81-95. doi:10.1007/ s10404-007-0222-z

95. Pradhan M, Pathak S, Mathur D, Ladiwala U. Optically Trapping Tumor Cells to Assess Differentiation and Prognosis of Cancers. Biomed Opt Express (2016) 7:943. doi:10.1364/BOE.7.000943

96. Xu W, Mezencev R, Kim B, Wang L, McDonald J, Sulchek T. Cell Stiffness Is a Biomarker of the Metastatic Potential of Ovarian Cancer Cells. PLoS ONE (2012) 7:e46609. doi:10.1371/journal.pone.0046609

97. Bendas G, Borsig L. Cancer Cell Adhesion and Metastasis: Selectins, Integrins, and the Inhibitory Potential of Heparins. Int J Cel Biol (2012) 2012:1-10. doi:10.1155/2012/676731

98. Casabella S, Scully P, Goddard N, Gardner P. Automated Analysis of Single Cells Using Laser Tweezers Raman Spectroscopy. Analyst (2016) 141:689-96. doi:10.1039/C5AN01851J

99. Qiu S, Weng Y, Li Y, Chen Y, Pan Y, Liu J, et al. Raman Profile Alterations of Irradiated Human Nasopharyngeal Cancer Cells Detected with Laser Tweezer Raman Spectroscopy. RSC Adv (2020) 10:14368-73. doi:10.1039/ D0RA01173H

100. Schaal F, Warber M, Zwick S, van der Kuip H, Haist T, Osten W. Marker-free Cell Discrimination by Holographic Optical Tweezers. J Eur Opt Soc (2009) 4. doi:10.2971/jeos.2009.09028

101. Li C-Y, Cao D, Qi C-B, Kang Y-F, Song C-Y, Xu D-D, et al. Combining Holographic Optical Tweezers with Upconversion Luminescence Encoding: Imaging-Based Stable Suspension Array for Sensitive Responding of Dual Cancer Biomarkers. Anal Chem (2018) 90:2639-47. doi:10.1021/acs.analchem.7b04299

102. Tomaiuolo G. Biomechanical Properties of Red Blood Cells in Health and Disease towards Microfluidics. Biomicrofluidics (2014) 8:051501. doi:10. $1063 / 1.4895755$

103. Lu T, Anvari B. Characterization of the Viscoelastic Properties of Ovarian Cancer Cells Membranes by Optical Tweezers and Quantitative Phase Imaging. Front Phys (2020) 8:582956. doi:10.3389/fphy.2020.582956

104. du Plooy JN. Comparison of Platelet Ultrastructure and Elastic Properties in Thrombo-Embolic Ischemic Stroke and Smoking Using Atomic Force and Scanning Electron Microscopy. PLoS ONE (2013) 8:e69774. doi:10.1371/ journal.pone.0069774

105. Hong-Lian G, Chun-Xiang L, Jian-Fa D, Yu-Qiang J, Xue-Hai H, Zhao-Lin L, et al. Mechanical Properties of Breast Cancer Cell Membrane Studied with Optical Tweezers. Chin Phys. Lett. (2004) 21:2543-6. doi:10.1088/0256-307X/21/12/062

106. Li X, Chen Z, Li Y, Liang H, Wang H, Li M. Optical Tweezers Study of Membrane Fluidity in Small Cell Lung Cancer Cells. Opt Express (2021) 29: 11976. doi:10.1364/OE.420288

107. Bustamante C, Alexander L, Maciuba K, Kaiser CM. Single-Molecule Studies of Protein Folding with Optical Tweezers. Annu Rev Biochem (2020) 89: 443-70. doi:10.1146/annurev-biochem-013118-111442

108. Scott MD, Frydman J. Aberrant Protein Folding as the Molecular Basis of Cancer. In: P Bross N Gregersen, editors. Protein Misfolding and DiseaseMethods in Molecular Biology ${ }^{\mathrm{TM}}$, 232 (2003). p. 1-67. doi:10.1385/1-59259-394-1Humana Press

109. Van Drie JH. Protein Folding, Protein Homeostasis, and Cancer. Chin J Cancer (2011) 30(2):124-37. doi:10.5732/cjc.010.10162

110. Knowles TPJ, Vendruscolo M, Dobson CM. The Amyloid State and its Association with Protein Misfolding Diseases. Nat Rev Mol Cel Biol (2014) 15: 384-96. doi:10.1038/nrm3810
111. Jiao J, Rebane AA, Ma L, Zhang Y. Single-Molecule Protein Folding Experiments Using High-Precision Optical Tweezers. In: A Gennerich, editor. Methods in Molecular Biology, 1486. New York: Humana Press (2017). p. 357-90. doi:10.1007/978-1-4939-6421-5_14

112. Bradonjić K, Swain JD, Widom A, Srivastava YN. The Casimir Effect in Biology: The Role of Molecular Quantum Electrodynamics in Linear Aggregations of Red Blood Cells. J Phys Conf Ser (2009) 161. doi:10.1088/ $1742-6596 / 161 / 1 / 012035$

113. Machta BB, Veatch SL, Sethna JP. Critical Casimir Forces in Cellular Membranes. Phys Rev Lett (2012) 109:138101. doi:10.1103/PhysRevLett. 109.138101

114. Ether DS, Pires LB, Umrath S, Martinez D, Ayala Y, Pontes B, et al. Probing the Casimir Force with Optical Tweezers. Epl (2015) 112:44001. doi:10.1209/ 0295-5075/112/44001

115. Callegari A, Magazzù A, Gambassi A, Volpe G. Optical Trapping and Critical Casimir Forces. Eur Phys J Plus (2021) 136:213. doi:10.1140/epjp/s13360020-01020-4

116. Zaltron A, Merano M, Mistura G, Sada C, Seno F. Optical Tweezers in SingleMolecule Experiments. Eur Phys J Plus (2020) 135:896. doi:10.1140/epjp/ s13360-020-00907-6

117. Zhang Y, Min C, Dou X, Wang X, Urbach HP, Somekh MG, et al. Plasmonic Tweezers: for Nanoscale Optical Trapping and beyond. Light Sci Appl (2021) 10:59. doi:10.1038/s41377-021-00474-0

118. Chen L, Liu W, Shen D, Zhou Z, Liu Y, Wan W. Label-free Plasmonic Assisted Optical Trapping of Single DNA Molecules. Opt Lett (2021) 46:1482. doi:10.1364/OL.420957

119. Pissuwan D, Valenzuela SM, Cortie MB. Therapeutic Possibilities of Plasmonically Heated Gold Nanoparticles. Trends Biotechnol (2006) 24: 62-7. doi:10.1016/j.tibtech.2005.12.004

120. Fitzgerald JM, Narang P, Craster RV, Maier SA, Giannini V. Quantum Plasmonics. Proc IEEE (2016) 104:2307-22. doi:10.1109/jproc.2016. 2584860

121. Kotnala A, Gordon R. Double Nanohole Optical Tweezers Visualize Protein P53 Suppressing Unzipping of Single DNA-Hairpins. Biomed Opt Express (2014) 5:1886. doi:10.1364/BOE.5.001886

122. Al Balushi AA, Kotnala A, Wheaton S, Gelfand RM, Rajashekara Y, Gordon R. Label-free Free-Solution Nanoaperture Optical Tweezers for Single Molecule Protein Studies. Analyst (2015) 140:4760-78. doi:10.1039/ C4AN02213K

123. Burkhartsmeyer J, Wang Y, Wong KS, GordonOptical Trapping, Sizing R, Acoustic P. Optical Trapping, Sizing, and Probing Acoustic Modes of a Small Virus. Appl Sci (2020) 10:394. doi:10.3390/app10010394

124. Ali MRK, Wu Y, El-Sayed MA. Gold-Nanoparticle-Assisted Plasmonic Photothermal Therapy Advances toward Clinical Application. J Phys Chem C (2019) 123:15375-93. doi:10.1021/acs.jpcc.9b01961

125. Huang X, El-Sayed MA. Plasmonic Photo-thermal Therapy (PPTT). Alexandria J Med (2011) 47:1-9. doi:10.1016/j.ajme.2011.01.001

126. Shan X, Wang F, Wang D, Wen S, Chen C, Di X, et al. Optical Tweezers beyond Refractive index Mismatch Using Highly Doped Upconversion Nanoparticles. Nat Nanotechnol (2021) 16:531-7. doi:10.1038/s41565-02100852-0

Conflict of Interest: The author declares that the research was conducted in the absence of any commercial or financial relationships that could be construed as a potential conflict of interest.

Publisher's Note: All claims expressed in this article are solely those of the authors and do not necessarily represent those of their affiliated organizations, or those of the publisher, the editors and the reviewers. Any product that may be evaluated in this article, orclaim that may be made by its manufacturer, is not guaranteed or endorsed by the publisher.

Copyright $\odot 2022$ Spyratou. This is an open-access article distributed under the terms of the Creative Commons Attribution License (CC BY). The use, distribution or reproduction in other forums is permitted, provided the original author $(s)$ and the copyright owner(s) are credited and that the original publication in this journal is cited, in accordance with accepted academic practice. No use, distribution or reproduction is permitted which does not comply with these terms. 